\title{
NUMERICAL STUDY OF DFT WITH MEAN SPHERICAL APPROXIMATION FOR IONIC CONDENSATION IN HIGHLY CHARGED CONFINED ELECTROLYTES
}

\author{
R. JOUBAUD ${ }^{1,2,3}$, O. BERNARD ${ }^{4,5}$, A. DELVILLE ${ }^{6}$, A. ERN ${ }^{2}$, B. ROTENBERG ${ }^{4,5}$, AND P. TURQ $^{5,4}$
}

\begin{abstract}
We investigate numerically a Density Functional Theory (DFT) for strongly confined ionic solutions in the Canonical Ensemble by comparing predictions of ionic concentration profiles and pressure for the double-layer configuration to those obtained with Monte Carlo (MC) simulations and the simpler Poisson-Boltzmann (PB) approach. The DFT consists of a bulk (ion-ion) and an ion-solid part. The bulk part includes nonideal terms accounting for long-range electrostatic and short-range steric correlations between ions and is evaluated with the Mean Spherical Approximation and the Local Density Approximation. The ion-solid part treats the ion-solid interactions at the mean-field level through the solution of a Poisson problem. The main findings are that ionic concentration profiles are generally better described by PB than by DFT, although DFT captures the non-monotone co-ion profile missed by $\mathrm{PB}$. Instead, DFT yields more accurate pressure predictions than $\mathrm{PB}$, showing in particular that nonideal effects are important to describe highly confined ionic solutions. Finally, we present a numerical methodology capable of handling nonconvex minimization problems so as to explore DFT predictions when the reduced temperature falls below the critical temperature.
\end{abstract}

\section{INTRODUCTION}

Charged solutes and charged interfaces are encountered in numerous natural and technological contexts, largely due to the ability of water to solvate ions and to screen their electrostatic interactions by its high dielectric permittivity. In this paper, we are interested in the study at the nanometric scale of ionic solutions interacting with (or confined by) solid objects carrying a surface charge (clay rocks, colloids, DNA, or electrodes, to name only a few examples). Multi-valent ions and electrolytes near such highly charged objects may depart significantly from the ideal behavior of infinitely diluted solutions.

In bulk situations, the nonideal behavior of ionic solutions arises mainly from two types of effects, which both play a larger role as the ionic concentration increases: long-range electrostatic interactions and short-range excluded volume effects. By treating the former at the mean-field level and ignoring the latter, the pioneering work of Debye and Hückel (DH) [1] identified screening by counterions as a fundamental mechanism for nonideality. The DH theory is valid at low concentrations and high temperature. For more concentrated solutions, further progress has been accomplished within the framework of the primitive models of charged hard spheres in a continuous solvent characterized by its dielectric constant, whereby structural and thermodynamic properties are predicted using integral equation theories, such as the Mean Spherical Approximation (MSA) [2, 3, 4, 5]. Liquid-vapor transition and criticality in bulk ionic solutions have been extensively investigated over the past decades. The properties of the liquid-vapor interface have been studied using a density-functional theory (DFT) within the restricted primitive model (RPM) in which the ions are modelled as equi-sized spheres 
carrying opposite charges [6]. Moreover, by combining Bjerrum's ion pairing concept [7] with the MSA or the DH theory through the law of mass action, better descriptions of the critical point and of the liquid-vapor coexistence curve have been obtained $[8,9,10,11,12]$.

In the case of confined ionic solutions, electrostatic interactions also control the structure and the phase behavior of the solution $[13,14,15]$. In some cases, such as clay minerals, cement, ion exchange membranes or lipid bilayers, the counterions compensating the charge of the surface may even be the only ions present in the confined fluid, resulting in a situation similar to the one-component plasma (see [16] for a review and [17] for a discussion closer to the present setting). The starting point for the description of confined ionic solutions is the Poisson-Boltzmann (PB) theory $[18,19,20]$. The PB theory ignores correlations between ions, and no phase transition exists within this approach, although quite high counterion densities can be predicted near charged surfaces in some conditions (e.g., cylindrical geometry $[21,22])$. For highly charged surfaces or multi-valent ions, a large fraction of the counterions appears condensed near the charged surface, as suggested by Stern to generalize the Gouy-Chapman description of charged surfaces. The remaining ions then feel a much weaker effective charge, which can be described within the PB theory. Nevertheless, the determination of the fraction of condensed ions and the corresponding renormalized charge is not straightforward; results in this direction can be found in [23]. Various approaches have been proposed to incorporate correlations neglected in the PB theory. In the particular case where counterions are the only ions present, a perturbative correction to the PB theory has been established [24]. Correlations for confined ions can also be included in integral theories, e.g., within the Anisotropic Hypernetted Chain approximation [25, 26]. Furthermore, the so-called "Strong Coupling" theory allows one to investigate regimes where the interaction with the charged surface is stronger than that between ions $[27,28,29,30,31]$ and to explain the origin of the attraction between like-charged surfaces observed under certain conditions [30, 32]. Another successful development for the description of the inhomogeneous primitive model is the use of DFT, which determines structural and thermodynamic properties of an inhomogeneous fluid from a Helmholtz free energy and its functional dependence on the local ionic densities [33, 34, 35, 36, 37, 38, 39, 40]. Finally, Molecular Dynamics and Monte Carlo simulations have been used to study the properties of bulk and confined electrolytes, described either within the primitive model or with an explicit molecular solvent, thus providing a more realistic description of these complex systems [41, 42, 43, 44, 45].

This paper's goal is to investigate numerically DFT predictions of confined ionic solutions at the nanometric scale by comparing ionic concentration profiles and pressure predictions to those obtained with MC and with the simpler PB approach for the double-layer configuration. We focus here on canonical constraints on the ionic densities fixing their mean value in the fluid domain while ensuring global electro-neutrality. In the present DFT, the Helmholtz free energy of the system depends on the ionic densities and splits into a bulk (ion-ion) and an ion-solid part. The former incorporates the ideal and nonideal effects in the bulk solution regardless of the presence of the charged solid object. The latter is based on a mean-field approach whereby the electrostatic potential is evaluated consistently with the ionic densities as the solution of a Poisson problem with Neumann boundary condition accounting for the surface charges carried by the solid object. Nonideal effects in the bulk free energy account for correlations between ions, including both long-range electrostatic and short-range steric contributions. These correlations are evaluated with the MSA and the Local Density Approximation (LDA) [46]. In particular, the screening length evaluated with the MSA depends on a nondimensional parameter which can be interpreted as a reduced temperature. To further 


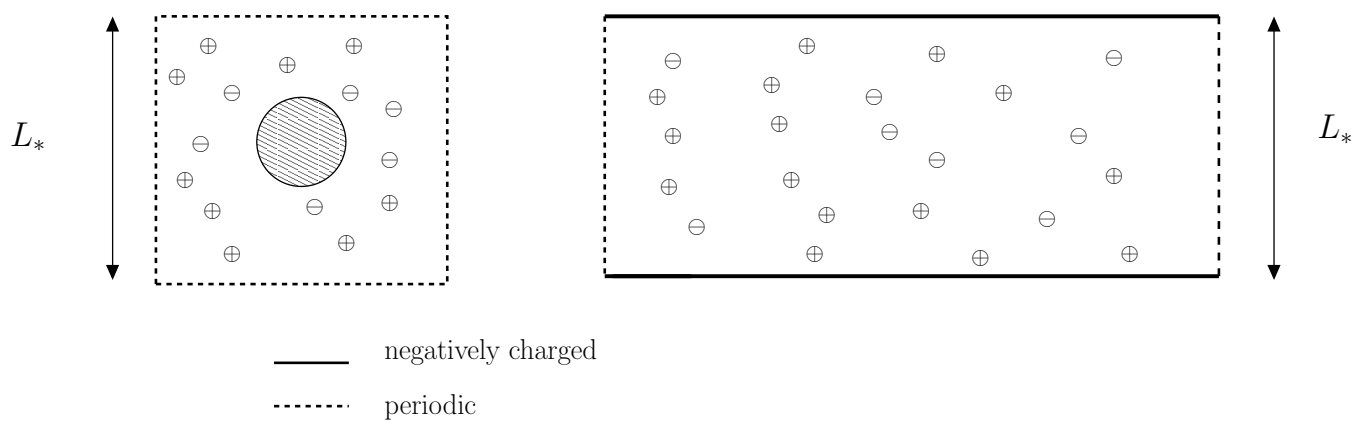

Figure 1. Geometric setting: elementary cell with charged inclusion (left); channel with charged walls (right).

understand the features of DFT-MSA predictions, we perform additional simulations where we consider only the long-ranged electrostatic contribution or the short-ranged steric contribution to the bulk free energy, and we also develop a robust numerical methodology to explore the situation where the reduced temperature falls below the critical temperature predicted by DFT-MSA, leading to phase separation.

The material is organized as follows. In Sect. 2, we present the DFT-MSA considered herein. In Sect. 3, we outline the numerical method for the constrained minimization within DFT and the more direct molecular approach based on MC simulations. In Sect. 4, we compare and analyze predictions obtained with DFT, MC, and PB. In Sect. 5, we present the numerical methodology to treat phase separation within DFT, and we discuss a few selected numerical results. Finally, in Sect. 6, we summarize our main findings and discuss further work.

\section{DFT FOR CONFINED IONIC SOLUTIONS}

We consider ionic solutions in the presence of solid objects carrying surface charges. Two typical settings are that of an ionic solution confined by charged walls and that of an ionic solution in a periodic setting with elementary cells containing a charged inclusion; see Fig. 1. While most of our results focus on the first setting, we briefly consider the more complex geometric setting of charged inclusions to illustrate the capabilities of the present numerical method. The length scale associated with the confinement is denoted by $L_{*}$ and is typically of the order of a nanometer. The domain occupied by the ionic solution is denoted by $\Omega$, and the domain occupied by the solid object is denoted by $\Omega_{\mathrm{S}}$. We assume that the solid object carries negative charges with surface density $-\Sigma_{\mathrm{S}}\left(\Sigma_{\mathrm{S}}>0\right)$ on its boundary $\partial \Omega_{\mathrm{S}}$.

For simplicity, we consider at most two dissolved ionic species, a counterion (cation) and a co-ion (anion). Ions are modelled within the primitive model as equi-sized spheres of diameter $\sigma$. The valences of the ionic species are denoted by $Z_{ \pm}$, and the case where $Z_{+}+Z_{-}=0$ is referred to as symmetric electrolyte (corresponding to the RPM). The ionic solution is described by the ionic (number) densities $\rho=\left(\rho_{+}, \rho_{-}\right)$in the fluid domain $\Omega$, while the solvent enters the model only by means of its relative dielectric permittivity. In what follows, we consider canonical constraints prescribing the mean-value of the ionic densities in the form

$$
\left\langle\rho_{ \pm}\right\rangle_{\Omega}=\rho_{ \pm}^{\text {mean }}
$$


where $\langle\cdot\rangle_{\Omega}$ denotes the mean-value of a function in the fluid domain $\Omega$. The prescribed quantities $\rho_{ \pm}^{\text {mean }}$ must satisfy the global electro-neutrality condition

$$
\sum_{i= \pm} Z_{i} \rho_{i}^{\text {mean }}=\frac{1}{e|\Omega|} \int_{\partial \Omega_{\mathrm{S}}} \Sigma_{\mathrm{S}}
$$

where $e$ is the elementary charge. A simple way of ensuring the global electro-neutrality condition (2) is to choose a density of added salt, $\rho^{\text {salt }}$, and to set

$$
\rho_{+}^{\text {mean }}=\rho_{+}^{\Sigma_{\mathrm{S}}}+\rho^{\text {salt }}, \quad \rho_{-}^{\text {mean }}=-\left(Z_{+} / Z_{-}\right) \rho^{\text {salt }},
$$

where $\rho_{+}^{\Sigma_{\mathrm{S}}}=\left(Z_{+} e|\Omega|\right)^{-1} \int_{\partial \Omega_{\mathrm{S}}} \Sigma_{\mathrm{S}}$ is the density of neutralizing counterions. In the vanishing limit of added salt, $\rho^{\text {salt }} \rightarrow 0$, we obtain an ionic solution with only compensating counterions.

The free energy functional of the ionic solution takes the form

$$
\mathcal{F}(\rho)=\mathcal{F}_{\text {bulk }}(\rho)+\mathcal{F}_{\mathrm{i}-\mathrm{s}}(\rho) .
$$

The bulk free energy functional $\mathcal{F}_{\text {bulk }}$ accounts for ideal and nonideal ion-ion interactions, while the functional $\mathcal{F}_{\mathrm{i}-\mathrm{s}}$ accounts for ion-solid interactions owing to the surface charges carried by the solid object. The functionals $\mathcal{F}_{\text {bulk }}$ and $\mathcal{F}_{\mathrm{i}-\mathrm{s}}$ are detailed in Sect. 2.1 and 2.2, respectively.

2.1. Bulk free energy functional. Within the LDA, the bulk free energy is obtained by integrating over the fluid domain $\Omega$ the bulk free energy density $f$, so that

$$
\mathcal{F}_{\text {bulk }}(\rho)=\int_{\Omega} f(\rho) \text {. }
$$

The bulk free energy density splits into an ideal part $f_{\text {id }}$ and a nonideal part $f_{\text {corr }}$ in the form

$$
f(\rho)=f_{\text {id }}(\rho)+f_{\text {corr }}(\rho), \quad f_{\text {corr }}(\rho)=f_{\text {Coul }}(\rho)+f_{\mathrm{HS}}(\rho),
$$

where $f_{\text {corr }}$ accounts for Coulomb electrostatic interactions (through $f_{\text {Coul }}$ ) and hard-sphere steric exclusion effects (through $f_{\mathrm{HS}}$ ). The ideal part reads

$$
f_{\text {id }}(\rho)=\frac{1}{\beta \sigma^{3}} \sum_{i= \pm} \hat{\rho}_{i}\left(\log \left(\hat{\rho}_{i}\right)-1\right)
$$

with nondimensional ionic densities $\hat{\rho}_{ \pm}=\sigma^{3} \rho_{ \pm}$, while $\beta=\left(k_{\mathrm{B}} T\right)^{-1}$ where $k_{\mathrm{B}}$ is the Boltzmann constant and $T$ the temperature. The Coulomb contribution is considered to be of the MSA form $[2,3,4,5]$

$$
f_{\mathrm{Coul}}(\rho)=-\frac{1}{4 \pi \beta \sigma^{3}}\left(\hat{\rho}_{\alpha}-4 \Gamma_{\mathrm{MSA}}\left(\hat{\rho}_{\alpha}\right)^{2}-\frac{16}{3} \Gamma_{\mathrm{MSA}}\left(\hat{\rho}_{\alpha}\right)^{3}\right),
$$

with $\hat{\rho}_{\alpha}=\left(\hat{\rho}_{+} / \alpha_{+}\right)+\left(\hat{\rho}_{-} / \alpha_{-}\right)$and the nondimensional parameters

$$
\alpha_{ \pm}=\frac{\sigma}{4 \pi L_{\mathrm{B}} Z_{ \pm}^{2}} .
$$

Here, $L_{\mathrm{B}}$ stands for the Bjerrum length given by $L_{\mathrm{B}}=(4 \pi \varepsilon)^{-1} \beta e^{2}$ where $\varepsilon=\varepsilon_{0} \varepsilon_{\mathrm{r}}$ with $\varepsilon_{0}$ the vacuum dielectric permittivity and $\varepsilon_{\mathrm{r}}$ the solvent relative dielectric permittivity. The parameters $\alpha_{ \pm}$are related to nondimensional temperatures $T_{ \pm}^{*}$ such that

$$
T_{ \pm}^{*}=4 \pi \alpha_{ \pm}=k_{\mathrm{B}} T \frac{\sigma(4 \pi \varepsilon)}{Z_{ \pm}^{2} e^{2}}
$$


while the inverse Debye length $\kappa$ is such that

$$
\kappa \sigma=\hat{\rho}_{\alpha}^{1 / 2} .
$$

The (nondimensional) screening parameter $\Gamma_{\mathrm{MSA}}$ is given by

$$
\Gamma_{\mathrm{MSA}}\left(\hat{\rho}_{\alpha}\right)=\frac{1}{2}\left(\left(2 \hat{\rho}_{\alpha}^{1 / 2}+1\right)^{1 / 2}-1\right) .
$$

Moreover, the hard-sphere contribution, which hinges on the Carnahan-Starling (CS) expression, is given by

$$
f_{\mathrm{HS}}(\rho)= \begin{cases}-\frac{6}{\pi \beta \sigma^{3}}\left(\frac{\xi\left(\hat{\rho}_{\mathrm{tot}}\right)^{2}\left(3 \xi\left(\hat{\rho}_{\mathrm{tot}}\right)-4\right)}{\left(1-\xi\left(\hat{\rho}_{\mathrm{tot}}\right)\right)^{2}}\right), & \xi\left(\hat{\rho}_{\mathrm{tot}}\right)<1, \\ +\infty, & \xi\left(\hat{\rho}_{\mathrm{tot}}\right) \geq 1,\end{cases}
$$

with reduced total ionic density $\hat{\rho}_{\text {tot }}=\hat{\rho}_{+}+\hat{\rho}_{-}$and packing number $\xi\left(\hat{\rho}_{\text {tot }}\right)=\frac{1}{6} \pi \hat{\rho}_{\text {tot }}$. In the dilute limit of low ionic densities, the CS expression can the linearized in the form (CS1)

$$
f_{\mathrm{HS}}(\rho)=\frac{1}{\beta \sigma^{3}} \frac{24}{\pi} \xi\left(\hat{\rho}_{\mathrm{tot}}\right)^{2} .
$$

A simplification occurs for symmetric electrolytes, since, in this case, $\alpha_{+}=\alpha_{-}=\alpha$ and $T_{+}^{*}=T_{-}^{*}=T^{*}$. Moreover, $\hat{\rho}_{\alpha}=\alpha^{-1} \hat{\rho}_{\text {tot }}$. As a result, $f_{\text {corr }}(\rho)$ only depends on $\hat{\rho}_{\text {tot }}$ and its properties can be described in terms of a single reduced temperature $T^{*}$.

2.2. Ion-solid free energy functional. The electrostatic interactions between ions and the solid object (as those between ions) are treated at the mean-field level. The electrostatic potential is computed consistently with the ionic densities by solving a Poisson problem in the fluid domain $\Omega$ with source term given by the charge density $q(\rho)=\sum_{i= \pm} Z_{i} e \rho_{i}$ and a Neumann boundary condition accounting for the surface charge carried by the solid object on $\partial \Omega_{\mathrm{S}}$. Specifically, the electrostatic potential $\psi(\rho)$ solves

$$
\left\{\begin{array}{l}
-\varepsilon \Delta \psi(\rho)=q(\rho) \quad \text { in } \Omega, \\
-\varepsilon \nabla \psi(\rho) \cdot \boldsymbol{n}=\Sigma_{\mathrm{S}} \quad \text { on } \partial \Omega_{\mathrm{S}}, \\
\psi(\rho) \text { periodic on } \partial \Omega \backslash \partial \Omega_{\mathrm{S}}, \\
\langle\psi(\rho)\rangle_{\Omega}=0,
\end{array}\right.
$$

where $\boldsymbol{n}$ denotes the unit outward normal to $\Omega$. The global electro-neutrality condition (2) ensures that problem (15) admits a solution, while the zero mean condition on $\psi(\rho)$ is just one possibility to fix the arbitrariness of $\psi(\rho)$ up to an additive constant. Finally, the functional $\mathcal{F}_{\mathrm{i}-\mathrm{s}}(\rho)$ is given by

$$
\mathcal{F}_{\mathrm{i}-\mathrm{S}}(\rho)=\frac{1}{2}\left(\int_{\Omega} q(\rho) \psi(\rho)-\int_{\partial \Omega_{\mathrm{S}}} \Sigma_{\mathrm{S}} \psi(\rho)\right)=\frac{\varepsilon}{2} \int_{\Omega}|\nabla \psi(\rho)|^{2},
$$

the second equality being a direct consequence of $(15)$ and integration by parts. Letting $G_{\Sigma_{\mathrm{S}}}$ denote the Green function of (15) with Dirac function as source term in $\Omega$, the boundary conditions remaining unchanged, the electrostatic potential can be rewritten as a convolution product in the form

$$
\psi(\rho)(x)=\int_{\Omega} G_{\Sigma_{\mathrm{S}}}(x, y) q(\rho)(y) d y
$$




\section{Numerical MEthods}

This section briefly describes the two numerical methods used in this work, namely the minimization of the free energy described in Sect. 2 and a more direct molecular approach based on Monte Carlo simulations.

3.1. Minimizing the free energy functional. Within the DFT, the equilibrium state of the ionic solution is determined by the following constrained minimization problem:

$$
\left\{\begin{array}{l}
\rho \text { minimizes } \mathcal{F}(\rho) \text { defined by }(4), \\
\rho \text { satisfies the canonical constraint }(1),
\end{array}\right.
$$

A (sufficient) well-posedness condition for (18) to have a unique solution hinges on the strict convexity of the bulk free energy density $f$ with respect to the ionic densities. This situation, which has been studied mathematically in [47] (using the linearized hard-sphere term (14)), is encountered when the reduced temperatures $T_{ \pm}^{*}$ are high enough (typically, above the critical temperature) so that the nonconvexity of the Coulomb interaction term $f_{\text {Coul }}$ is compensated by the convexity of the ideal and hard-sphere terms $f_{\text {id }}$ and $f_{\mathrm{HS}}$. For a symmetric electrolyte where the function $f$ is univariate, a study of the convexity of this function shows that the critical temperature and (total) density are $T_{\text {crit }}^{*} \approx 0.0785$ and $\hat{\rho}_{\text {tot,crit }} \approx 0.0145$ (see Fig. 7 below), in agreement with the result previously derived in [6]. As is well-known, the MSA overpredicts the critical temperature, which is about $T_{\text {crit }}^{*}=0.05$ according to Monte Carlo simulations [48]. For non-symmetric electrolytes, a study of the convexity of the bulk free energy functional can be found in [49].

The Euler-Lagrange equations associated with (18) are formulated in mixed form by introducing as an additional unknown the electrostatic potential $\psi=\psi(\rho)$ solving (15). The constraints to be taken into account are the canonical constraint (1) on the ionic densities $\rho$ and the fact that $\psi$ has zero mean value in $\Omega$. In view of finite element discretization, we write the Euler-Lagrange equations in variational form using test functions. Thus, we seek ionic densities $\rho$, electrostatic potential $\psi$, and real numbers $\lambda$ and $\mu_{ \pm}^{\text {bulk }}$ such that

$$
\left\{\begin{array}{l}
\int_{\Omega} \varepsilon \nabla \psi \cdot \nabla \varphi+\lambda\langle\varphi\rangle_{\Omega}=\int_{\Omega} q(\rho) \varphi-\int_{\partial \Omega_{\mathrm{S}}} \Sigma_{\mathrm{S}} \varphi \\
\int_{\Omega} \mu_{ \pm}^{\mathrm{el}}(\rho, \psi) v_{ \pm}=\mu_{ \pm}^{\mathrm{bulk}}|\Omega|\langle v\rangle_{\Omega}, \\
r\langle\psi\rangle_{\Omega}=0, \quad s_{ \pm}\left(\left\langle\rho_{\eta, \pm}\right\rangle_{\Omega}-\rho_{ \pm}^{\text {bulk }}\right)=0
\end{array}\right.
$$

for all test functions $\varphi$ and $v_{ \pm}$and for all real numbers $r$ and $s_{ \pm}$. The electro-chemical potential $\mu_{ \pm}^{\mathrm{el}}(\rho, \psi)$ is such that

$$
\mu_{ \pm}^{\mathrm{el}}(\rho, \psi)=\mu_{ \pm}(\rho)+Z_{ \pm} e \psi
$$

with chemical potential

$$
\mu_{ \pm}(\rho)=\partial_{\rho_{ \pm}} f(\rho) .
$$

The second equation in (19) expresses the fact that the electro-chemical potentials are constant in $\Omega$ (with constant value equal to $\mu_{ \pm}^{\text {bulk}}$ ).

The Euler-Lagrange equations (19) are discretized using finite elements. The electrostatic potential and the ionic densities are approximated using continuous, piecewise affine functions over a mesh of the fluid domain $\Omega$. This leads to a (large) system of nonlinear algebraic 
equations which is solved iteratively using a Newton-Raphson algorithm. Each NewtonRaphson iteration consists in assembling a linear system whose solution provides an update for the discrete solution vector. By linearity, at each iteration, the electrostatic potential is evaluated consistently with the ionic densities, and the constraints on the mean value of both the electrostatic potential and the ionic densities are satisfied. A clipping is applied to the update vector resetting to the interval $[\epsilon, 6 / \pi-\epsilon]$ with small $\epsilon$ any ionic density that may have fallen outside this interval during the convergence process, while the ionic densities at the other mesh nodes are rescaled in agreement with the canonical constraint (1). The convergence of the Newton-Raphson algorithm is monitored by checking the Euclidean norm of the discrete residual vector and that of the update vector at each iteration. Typically, convergence is achieved after 15 to 20 iterations, and the is usually needed only at (very) few iterations. More details on the algorithmic aspects can be found in [50].

3.2. Monte Carlo simulations. Monte Carlo simulations are performed in the Canonical Ensemble to determine the equilibrium distribution of the di- and trivalent ions confined between two charged planes. In each case, the simulation cell is maintained electrically neutral, a condition necessary to reach the thermodynamic limit, i.e., to obtain results independent of the size of the simulation cell. The limiting charged lamellae are structure-less and infinite, with a uniform surface charge density. In the framework of the primitive model, ion-ion and ion-lamella interactions include short-ranged contact repulsion and long-ranged Coulomb potential. The solvent is replaced by a continuum characterized by its relative dielectric permittivity $\varepsilon_{\mathrm{r}}$, equal to the relative lamellar dielectric permittivity.

The simulation cell is an asymmetric slit pore, limited in the longitudinal direction by the charged lamellae while periodic boundary conditions are applied in the lateral direction. Since 2D minimum image convention is applied in the transverse directions, the ion-ion, ion-lamella and lamella-lamella electrostatic interactions are cut at the half-width of the simulation cell. In order to reproduce the long-range of the Coulomb potential, an external potential is introduced in a self-consistent manner [51, 52, 53]. This external electrostatic potential is generated by a supplementary set of infinite planes parallel to the two confining charged lamellae. The uniform surface charge density of these supplementary lamellae is evaluated in a self-consistent manner from the ionic local densities in the simulation cell. A square hole, corresponding to the lateral section of the simulation cell, is cut within each supplementary lamella to remove the contribution from the simulation cell. The convergence of that approximation is carefully checked by doubling the lateral extent of the simulation cell [53]. In the present case, lateral extents of 200 and $400 \AA$ are used successfully for charged interfaces neutralized by divalent and trivalent ions, respectively. Furthermore, three independent and consecutive sets of, at least, $5 \times 10^{8}$ iterations are performed in order to ensure the convergence of the self-consistent procedure. This method has been applied in the literature to investigate the stability of a large class of charged interfaces $[54,55,56]$.

To evaluate the pressure, we proceed as follows. After thermalization of the ionic concentration profiles, the longitudinal component of the pressure tensor is evaluated locally at half separation between the charged limiting surfaces. Such a procedure is valid since, at equilibrium, the longitudinal component of the pressure is constant along the whole interfacial system. In addition to the repulsive entropic contribution evaluated from the local densities of the confined ions, the primitive model includes two additional contributions resulting from the long-range electrostatic couplings and the short-range ion-ion steric repulsions. These 
two additional contributions result from the ionic correlations leading respectively to antagonistic contributions, i.e., a net attraction for the electrostatic coupling and, obviously, a net repulsion for the ion-ion contact forces. These two components are evaluated by summing the longitudinal component of the net forces transmitted across a fictitious plane surface $[55,56]$.

\section{Results AND Discussion}

In this section we investigate the predictions of DFT-MSA for the ionic density profiles and the pressure, under severe confinement conditions. The results are compared to the reference Monte Carlo (MC) simulations, as well as to the simpler Poisson-Boltzmann (PB) model. In what follows, we consider a confining distance $L_{*}=1 \mathrm{~nm}$ (determining the accessible volume) and discuss the influence of the surface charge density $\Sigma_{\mathbf{S}}$, the ion diameter $\sigma$, and salt concentration $\rho^{\text {salt }}$ on the ionic profiles and the pressure. We also consider the effect of the relative permittivity $\varepsilon_{\mathrm{r}}$ and of the valences $Z_{ \pm}$for a symmetric electrolyte. In all cases, the reduced temperature $T^{*}$ defined by $(10)$ is in the range $[0.08,0.16]$, i.e., above the critical point (but not too far from it). In order to understand the origin of the features observed with DFT-MSA, we finally consider separately the effects of the hard sphere and electrostatic correlation contributions to the functional.

4.1. Divalent ions: density profiles. We first examine ionic density profiles for divalent ions $\left(Z_{ \pm}= \pm 2\right)$, for fixed relative permittivity $\varepsilon_{\mathrm{r}}=78.5$ and salt concentration $\rho^{\text {salt }}=$ $0.5 \mathrm{~mol} \mathrm{~L}^{-1}$. Fig. 2(a) reports the counter- and co-ions density profiles for a surface charge density $\Sigma_{\mathrm{S}}=0.2 \mathrm{C} \mathrm{m}^{-2}$ and ion diameter $\sigma=2.55 \AA\left(T^{*}=0.09\right)$. For both profiles, the MC results are better described by PB than by DFT-MSA, in particular near the charged surface where DFT-MSA overestimates the densities. However, the co-ion density is non-monotonous and displays a secondary minimum at the center of the pore. This feature is not reproduced by PB, while DFT-MSA is able to do so. The position of the maximum is nevertheless too close to the surface.

Increasing the surface charge density to $\Sigma_{\mathrm{S}}=0.6 \mathrm{C} \mathrm{m}^{-2}$, as shown in Fig. $2 \mathrm{~b}$, we find that DFT-MSA now underestimates the counterion density at contact. However, DFT-MSA still predicts the observed non-monotonous co-ion profile missed by PB. Comparing the MC co-ion concentration profiles between panels (a) and (b), we observe that the position of the maximum slightly changes, while the peak is more pronounced; the density near the surface and in the center of the pore are reduced accordingly. With DFT-MSA, the position of the peak shifts away from the surface, thereby approaching the MC results. The overestimation of the co-ion density near the surface has now disappeared, and the DFT-MSA prediction is now in very good agreement with the MC results, while a sizable difference remains at the center of the pore.

We now consider, for $\Sigma_{\mathrm{S}}=0.2 \mathrm{C} \mathrm{m}^{-2}$, an increase in the ion diameter to $\sigma=4.54 \AA$ $\left(T^{*}=0.16\right)$, as shown in Fig. 2c. While the counterion profile is still better described by PB, DFT-MSA slightly better reproduces the co-ion density. In this regime, the profile increases monotonically from the surface to the center. Compared to PB, DFT-MSA predicts a lower density near the surface and a slightly higher density near the center, i.e., moving in the direction of the MC results. Finally, Fig. 2d reports the results for the same surface charge density and $\sigma=2.27 \AA\left(T^{*}=0.08\right)$. In this case, the failure of DFT-MSA is dramatic, with a large density of both counter- and co-ions near the surface and accordingly low densities 


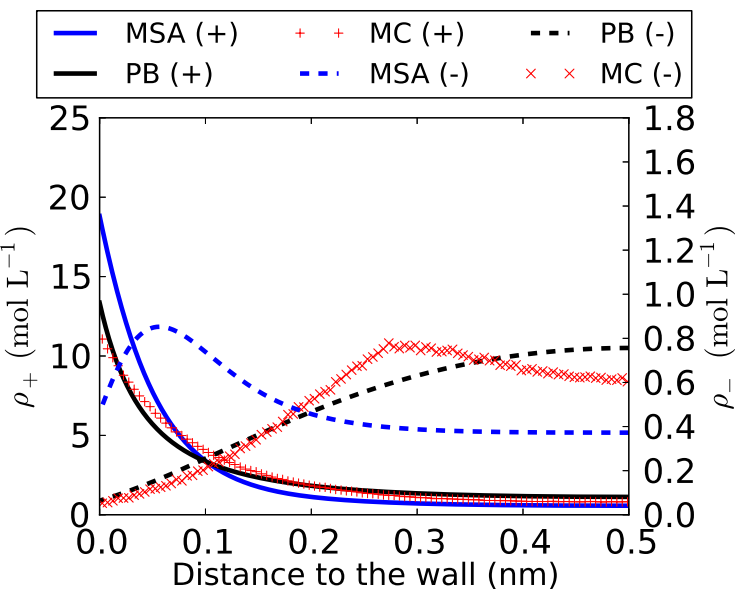

a

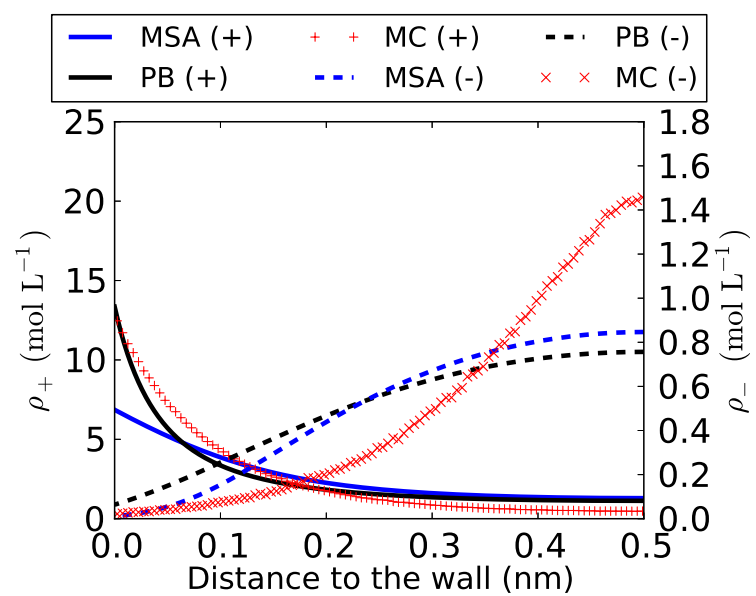

C

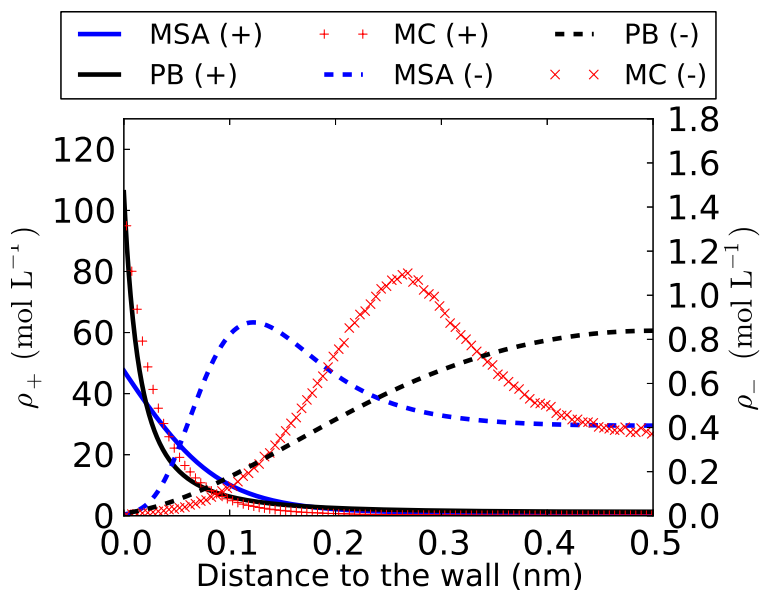

b

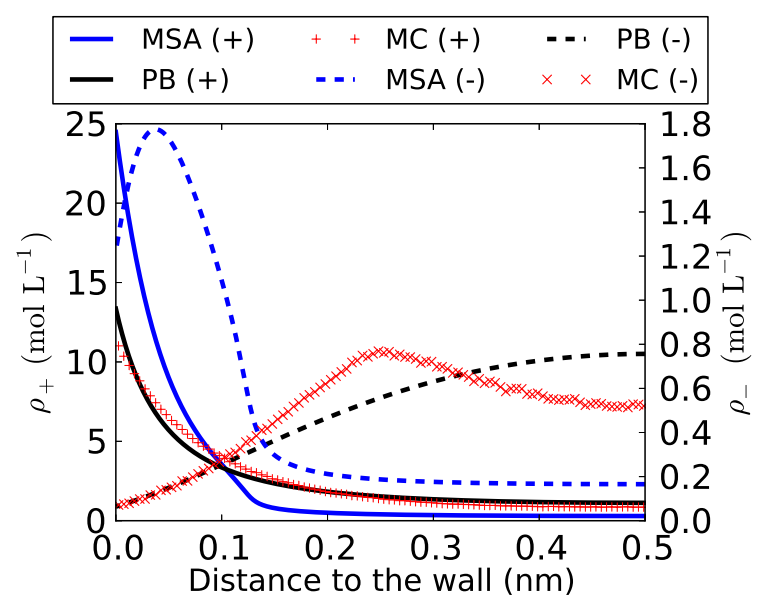

d

Figure 2. (Color online) Density profiles for divalent ions $\left(Z_{ \pm}= \pm 2\right.$ ), relative permittivity $\varepsilon_{\mathrm{r}}=78.5$, and salt concentration $\rho^{\text {salt }}=0.5 \mathrm{~mol} \mathrm{~L}^{-1}$. (a) Surface charge density $\Sigma_{\mathrm{S}}=0.2 \mathrm{C} \mathrm{m}^{-2}$ and ion diameter $\sigma=2.55 \AA$ ( $\left.T^{*}=0.09\right)$. (b) $\Sigma_{\mathrm{S}}=0.6 \mathrm{C} \mathrm{m}^{-2}$ and $\sigma=2.55 \AA\left(T^{*}=0.09\right)$. (c) $\Sigma_{\mathrm{S}}=0.2 \mathrm{C} \mathrm{m}^{-2}$ and $\sigma=$ $4.54 \AA\left(T^{*}=0.16\right)$. (d) $\Sigma_{\mathrm{S}}=0.2 \mathrm{C} \mathrm{m}^{-2}$ and $\sigma=2.27 \AA\left(T^{*}=0.08\right)$. Solid lines and + indicate counterion profiles, while dashed lines and $\times$ correspond to co-ions. DFT-MSA (blue) is compared to Poisson-Boltzmann (black) and Monte Carlo (symbols) results.

near the center. This behavior is likely due to an incipient phase transition as we approach the critical point. This aspect is further examined in Sect. 5.

4.2. Divalent ions: pressure. We now turn to the study of the pressure. The pressure from MC calculations is evaluated as described in Sect. 3.2. The pressure from DFT-MSA 
and PB calculations is evaluated at the center of the pore using the Gibbs-Duhem rules

$$
p(\rho)=\rho_{+} \mu_{+}(\rho)+\rho_{-} \mu_{-}(\rho)-f(\rho) .
$$

For completeness, we also evaluate the pressure in the bulk reservoir in thermodynamic equilibrium with the confined system by evaluating the bulk concentration corresponding to a system at the same chemical potentials $\mu_{ \pm}^{\text {bulk. }}$. As the concentration in the bulk have to be equal, we introduce a shift $\zeta$ (consistently with our approach for the confined system where we have fixed the mean of the electrostatic potential arbitrarily to 0 ), so that the two equations satisfied by the densities in the bulk are

$$
\mu_{ \pm}\left(\rho^{\text {bulk }}\right)+Z_{ \pm} \zeta=\mu_{ \pm}^{\text {bulk }}
$$

These equations can be solved by a Newton-Raphson algorithm for each value of the shift parameter $\zeta$, and the actual value of this parameter, which is such that the solution to (23) verifies $\rho_{+}^{\text {bulk }}=\rho_{-}^{\text {bulk }}$, is found by dichotomy.

Starting from the same system as in Fig. 2a, namely $Z_{ \pm}= \pm 2, \varepsilon_{\mathrm{r}}=78.5, \rho^{\text {salt }}=$ $0.5 \mathrm{~mol} \mathrm{~L}{ }^{-1}, \Sigma_{\mathrm{S}}=0.2 \mathrm{C} \mathrm{m}^{-2}$, and $\sigma=2.55 \AA\left(T^{*}=0.09\right)$, we change $T^{*}$ by increasing $\sigma$ up to $4.54 \AA\left(T^{*}=0.16\right)$. Results are reported in Fig. 3a. MC simulations indicate that the pressure increases quadratically with $T^{*}$. PB does not capture the evolution with ion diameter and overestimates the pressure in this range. In contrast, DFT-MSA accurately describes the change in pressure with $T^{*}$ even though its predictions are shifted (by an almost constant value in this range) from the MC results. The PB and DFT-MSA pressures for bulk electrolytes in thermodynamic equilibrium with the confined system are also displayed and found to be almost identical to the pressures under confinement. The effect of the surface charge density can be seen in Fig. 3b. Increasing $\Sigma_{\mathrm{S}}$ to $0.4 \mathrm{C} \mathrm{m}^{-2}$, we observe that the MC pressure decreases at low $T^{*}$, down to negative values for $T^{*} \leq 0.11$, and increases for the largest values of $T^{*}$. While the constant PB result increases with $\Sigma_{\mathrm{S}}$, DFT-MSA better reproduces the quadratic variation predicted by $\mathrm{MC}$ simulations.

In Fig. $4, T^{*}$ is changed by increasing the relative permittivity $\varepsilon_{\mathrm{r}}$ for a fixed ion diameter $\sigma$. The pressure increases almost linearly with $T^{*}$. The PB pressure now also increases, but with too small a slope and starting from a much larger value. Instead, DFT-MSA is almost in quantitative agreement with $\mathrm{MC}$ for a salt concentration $\rho^{\text {salt }}=0.5 \mathrm{~mol} \mathrm{~L}-1$. Here again, the pressure in the confined system is almost identical to that in the bulk reservoir with which it is in equilibrium. In the absence of salt, the pressure decreases for MC, DFT-MSA, and PB. MC simulations indicate that it even becomes negative for $T^{*} \leq 0.11$, a feature that is not reproduced by either PB or DFT-MSA. The deviations of DFT-MSA from MC results are much larger than in the presence of salt, but decrease as $T^{*}$ increases.

4.3. Trivalent ions. We consider now trivalent ions $\left(Z_{ \pm}= \pm 3\right)$ at the same ionic strength as for the above divalent ions, leading to a salt concentration $\rho^{\text {salt }}=0.2222 \mathrm{~mol} \mathrm{~L}^{-1}$, in a solvent with relative permittivity $\varepsilon_{\mathrm{r}}=78.5$ and ion diameter $\sigma=5.1 \AA\left(T^{*} \approx 0.08\right.$ (slightly) above the critical point). The surface charge density is $\Sigma_{\mathrm{S}}=0.2 \mathrm{C} \mathrm{m}^{-2}$. The ionic density profiles are reported in Fig. 5a. The MC results are qualitatively similar to the ones for divalent ions in this regime (see Fig. 2c). The predictions of DFT-MSA are worse than those of $\mathrm{PB}$ for both counter- and co-ions, except for the stronger exclusion of co-ions very close to the surface. The pressure, reported in Fig. $5 \mathrm{~b}$ as a function of $T^{*}$ (changed by increasing $\varepsilon_{\mathrm{r}}$ ), behaves as in the divalent case. Despite its poorer prediction of the ionic profiles, DFT-MSA reproduces much better than $\mathrm{PB}$ the pressure predicted by MC. 


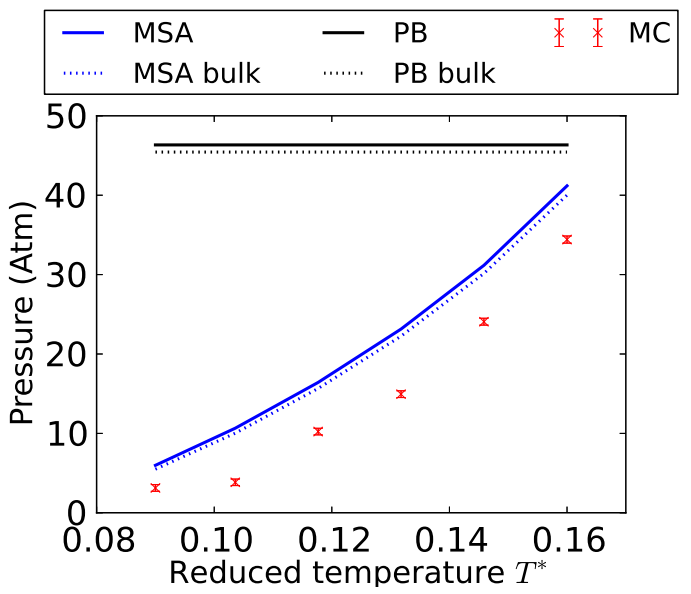

a

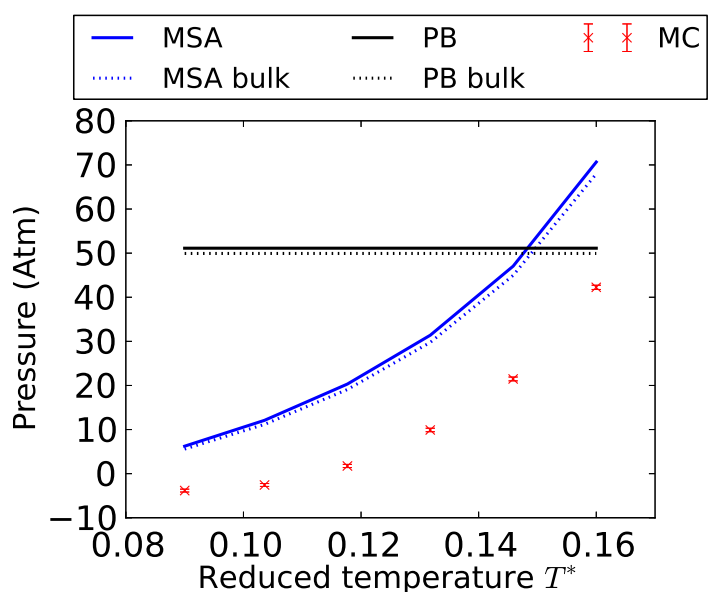

$\mathrm{b}$

Figure 3. (Color online) Pressure for divalent ions $\left(Z_{ \pm}= \pm 2\right.$ ), relative permittivity $\varepsilon_{\mathrm{r}}=78.5$, and salt concentration $\rho^{\text {salt }}=0.5 \mathrm{~mol} \mathrm{~L}{ }^{-1}$, as a function of reduced temperature $T^{*}$ changed by increasing the ion diameter $\sigma$ from left to right, for a surface charge density $\Sigma_{\mathrm{S}}=0.2$ (a) and $0.4 \mathrm{C} \mathrm{m}^{-2}$ (b). DFT-MSA (blue) is compared to Poisson-Boltzmann (black) and Monte Carlo (symbols) results. Dashed lines indicate the DFT-MSA and PB results in the bulk in thermodynamic equilibrium with the system.

4.4. Hard sphere and electrostatic contributions. We finally investigate the origin of the features observed on the ionic density profiles within DFT-MSA. Compared to PB, the MSA free energy functional includes two additional contributions: the hard sphere (hs) volume exclusion term defined in (8) and the electrostatic correlation (el) term defined in (13). In order to assess their effects on the full MSA solution, we consider two additional cases, where we include only one or the other term in the functional. Results for counter- and co-ions are reported in Figs. $6 \mathrm{a}$ and $6 \mathrm{~b}$, respectively, for divalent ions $\left(Z_{ \pm}= \pm 2\right), \Sigma_{\mathrm{S}}=0.2 \mathrm{C} \mathrm{m}^{-2}$, $\rho^{\text {salt }}=0.5 \mathrm{~mol} \mathrm{~L}^{-1}, \sigma=2.55 \AA$, and $\varepsilon_{\mathrm{r}}=78.5\left(T^{*}=0.09\right)$, i.e., the same situation as Fig. $2 \mathrm{a}$.

Adding only the hard sphere term to PB has almost no effect on the co-ions, but slightly decreases the counterion concentration near the surface, as expected, thereby providing a better agreement with the MC results. On the contrary, adding only the electrostatic correlations (at the MSA level) results under the present conditions in a wild overestimate of both densities near the surface. In the absence of hard core repulsion, the chosen $T^{*}$ probably corresponds in the bulk to a phase coexistence, and the separation between a high density (close to the surface) and a low density (near the center) region is reminiscent of this transition. Even though this pure electrostatic correction is not relevant to the description of the real system, it suggests that the full DFT-MSA overestimate of the counterion density near the surface can be traced back to this contribution. In addition, these electrostatic correlations are at the origin of the non-monotonous behavior of the co-ion density (when it occurs). Finally, since the hard sphere contribution can only increase the pressure, the electrostatic correlation term is responsible for the smaller pressure with DFT-MSA than with PB, resulting in the better agreement with MC results. 


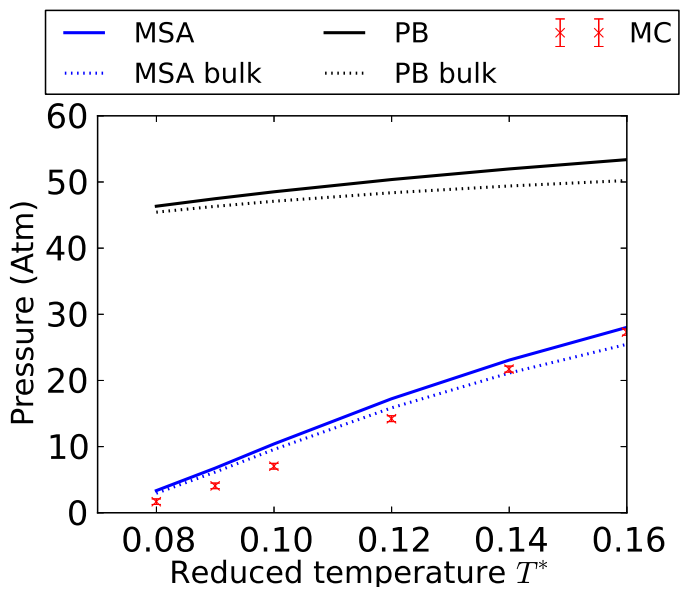

a

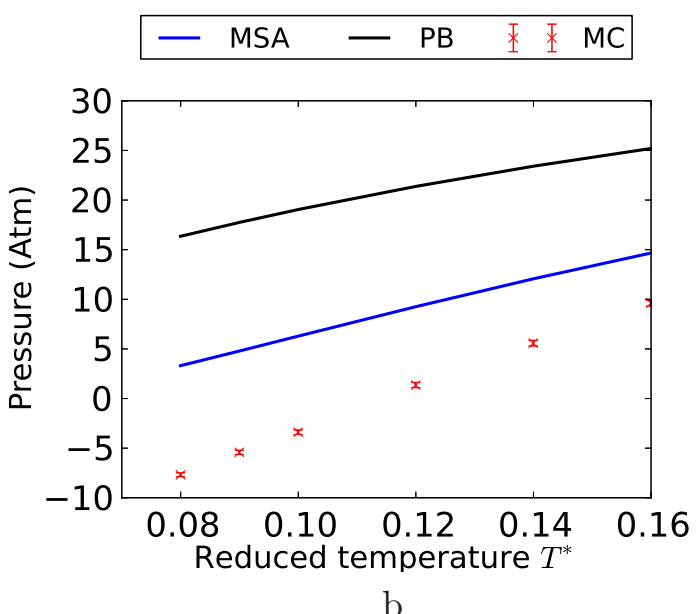

$\mathrm{b}$

Figure 4. (Color online) Pressure for divalent ions $\left(Z_{ \pm}= \pm 2\right.$ ), surface charge density $\Sigma_{\mathrm{S}}=0.2 \mathrm{C} \mathrm{m}^{-2}$, and ion diameter $\sigma=2.27 \AA$, as a function of reduced temperature $T^{*}$ changed by increasing the relative permittivity $\varepsilon_{\mathrm{r}}$ from left to right, for salt concentration $\rho^{\text {salt }}=0.5$ (a) and $0 \mathrm{~mol} \mathrm{~L}^{-1}$ (b). DFT-MSA (blue) is compared to Poisson-Boltzmann (black) and Monte Carlo (symbols) results. Dashed lines indicate the DFT-MSA and PB results in the bulk in thermodynamic equilibrium with the system.

\section{Phase separation Within DFT-MSA}

This section briefly examines the present DFT-MSA when the bulk free energy density is no longer convex with respect to the ionic densities. We recall that in the case of bulk ionic solutions, this regime leads to liquid-vapor transition. We emphasize that the present DFT-MSA results substantially differ from MC predictions.

5.1. The constrained minimization problem. In confined ionic solutions, the presence of the charged solid object induces gradients in the ionic densities and, thereby, departures from local electro-neutrality condition $\sum_{i= \pm} Z_{i} e \rho_{i}=0$. As a result, the whole state space $\mathcal{K}:=\left\{\rho \in \mathbb{R}^{2} ; \rho_{ \pm} \geq 0 ; \xi(\hat{\rho})<1\right\}$ can potentially be explored by the ionic densities at different points in $\Omega$. Letting $f^{* *}$ be the convex hull of $f$, we introduce the subset

$$
\mathcal{K}^{* *}=\left\{\rho \in \mathcal{K} ; f(\rho)=f^{* *}(\rho)\right\},
$$

and we supplement the constrained minimization problem (18) with the additional constraint that, everywhere in $\Omega$, the ionic densities take values in the subset $\mathcal{K}^{* *}$. This constraint hinges on the assumption that the bulk system should be thermodynamically stable regardless of confinement; this is why the convexification is performed only on the bulk part of the free energy functional. The constrained minimization problem can be stated as follows:

$$
\left\{\begin{array}{l}
\rho \text { minimizes } \mathcal{F}(\rho) \text { defined by }(4), \\
\rho \text { satisfies the canonical constraint }(1), \\
\text { For all } x \in \Omega, \rho(x) \text { takes values in } \mathcal{K}^{* *} \text { defined by }(24) .
\end{array}\right.
$$




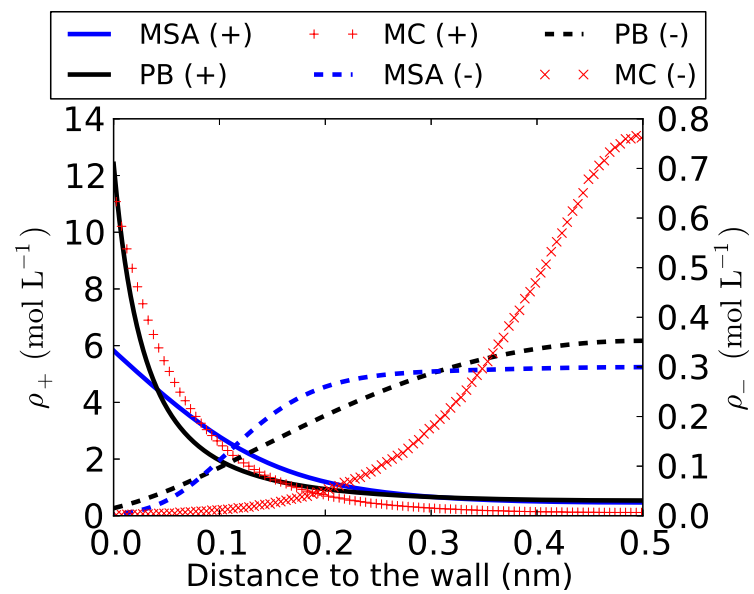

a

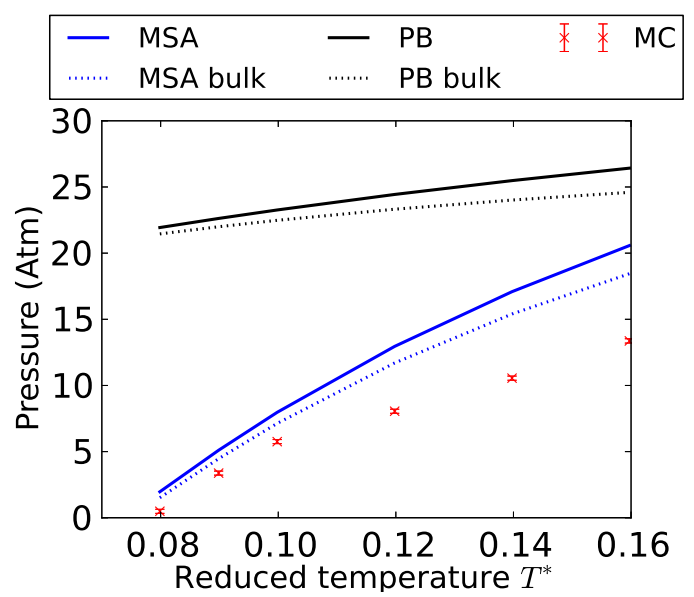

$\mathrm{b}$

Figure 5. (Color online) Trivalent ions $\left(Z_{ \pm}= \pm 3\right.$ ) with ion diameter $\sigma=$ $5.1 \AA$, salt concentration $\rho^{\text {salt }}=0.2222 \mathrm{~mol} \mathrm{~L}^{-1}$ and surface charge density $\Sigma_{\mathrm{S}}=0.2 \mathrm{C} \mathrm{m}^{-2}$. (a) Ionic density profiles for a relative permittivity $\varepsilon_{\mathrm{r}}=78.5$ $\left(T^{*} \approx 0.08\right)$; see Fig. 2 for legends. (b) Pressure as a function of reduced temperature $T^{*}$ changed by increasing the relative permittivity $\varepsilon_{\mathrm{r}}$ from left to right; see Fig. 3 for legends.

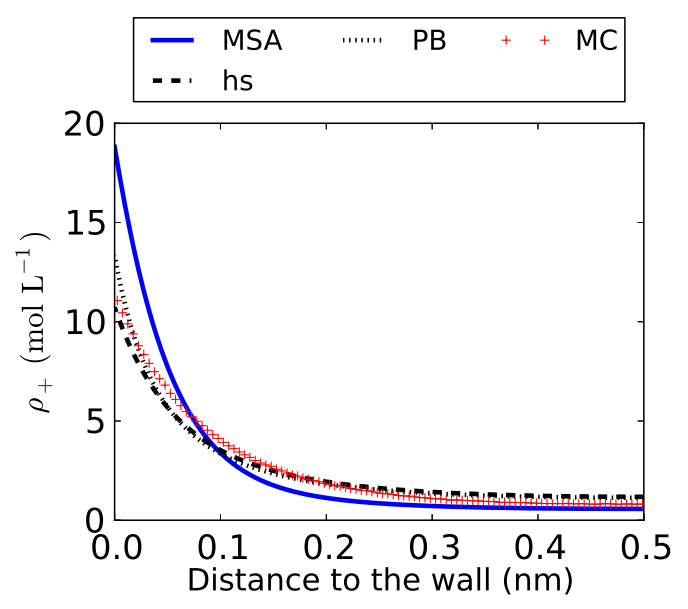

a

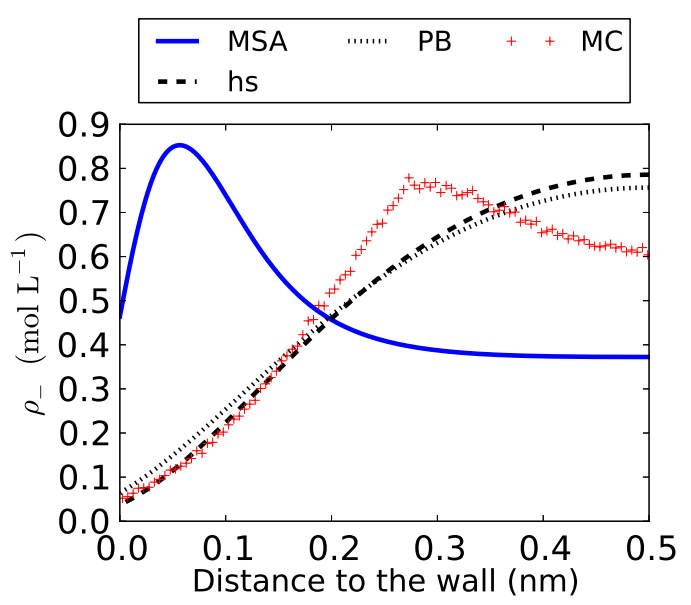

$\mathrm{b}$

Figure 6. (Color online) Counterion (a) and co-ion (b) density profiles for divalent ions $\left(Z_{ \pm}= \pm 2\right)$, surface charge density $\Sigma_{\mathrm{S}}=0.2 \mathrm{C} \mathrm{m}^{-2}$, salt concentration $\rho^{\text {salt }}=0.5 \mathrm{~mol} \mathrm{~L}^{-1}$, ion diameter $\sigma=2.55 \AA$, and relative permittivity $\varepsilon_{\mathrm{r}}=78.5\left(T^{*}=0.09\right)$. Monte Carlo results $($ red $\times)$ are compared to PB (dotted black line), full DFT-MSA (solid blue line) and DFT with only the hard-sphere contribution (hs, black dashed line).

The mathematical analysis of (25) goes beyond the present scope. There is an extensive mathematical (and numerical) bibliography on phase separation in other settings than confined electrolytes. In general, the functional used in such phase-field theory is a double-well 
potential inducing a partition of the state space into two phases. Most of the studies have been performed in bulk situations where the state variable (here, the ionic densities) is constant in each phase. The key idea is to regularize the problem by minimizing a regularized functional where a least-squares penalty on the gradient of the state variable is added to the double-well potential. For the mathematical analysis showing that the limit minimization problem as the regularization parameter tends to zero involves the convex hull of the double-well potential and that this limit represents a choice criterion among minimizers of the original nonconvex variational problem so as to minimize interfaces by sampling states where the potential and its convex hull coincide, we refer, e.g., to the seminal work in [57]; further references and links to $\Gamma$-convergence can be found in [58]. The situation where the double-well potential is perturbed by, e.g., a nonlocal operator is more delicate to analyze; we mention [59, 60], and, more recently, [61] where a nonlocal operator accounting for Coulomb interaction is added.

In the case of symmetric electrolytes, it is possible to approximate with very good accuracy the subset $\mathcal{K}^{* *}$. To this purpose, we write $f(\rho)=f_{\text {id }}(\rho)+f_{\text {corr }}\left(\rho_{\text {tot }}\right)$ exploiting the fact that $f_{\text {corr }}$ only depends on $\rho_{\text {tot }}$. Re-arranging the ideal part leads to $f(\rho)=f_{\text {tot }}\left(\rho_{\text {tot }}\right)+f_{\text {rel }}(\rho)$, where

$$
f_{\text {tot }}\left(\rho_{\text {tot }}\right)=\frac{1}{\beta \sigma^{3}} \hat{\rho}_{\text {tot }}\left(\log \left(\hat{\rho}_{\text {tot }}\right)-1\right)+f_{\text {corr }}\left(\rho_{\text {tot }}\right),
$$

and

$$
f_{\text {rel }}(\rho)=\frac{1}{\beta \sigma^{3}} \sum_{i= \pm} \hat{\rho}_{i} \log \left(\frac{\hat{\rho}_{i}}{\hat{\rho}_{\text {tot }}}\right) .
$$

The bivariate function $f_{\text {rel }}$ is convex in the ionic densities, and the subset $\mathcal{K}^{* *}$ is approximated by studying the convexity properties of the univariate function $f_{\text {tot }}$ (i.e., we assume that $\left.f^{* *} \sim f_{\text {rel }}+f_{\text {tot }}^{* *}\right)$.

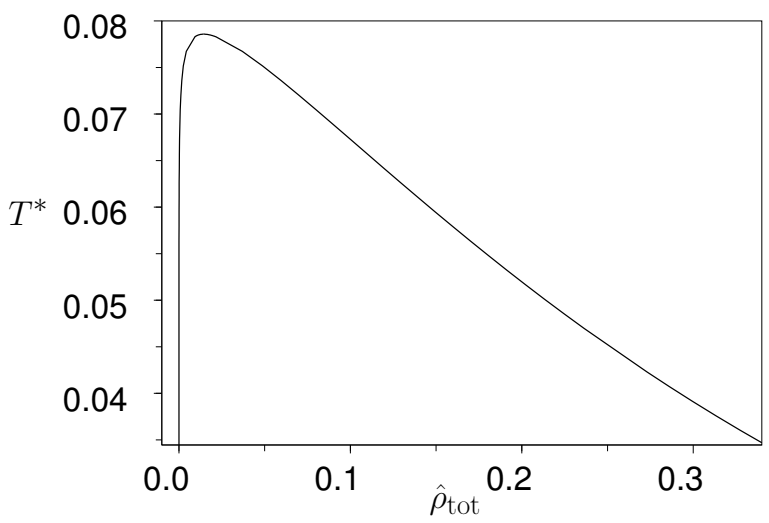

Figure 7. Phase diagram for a bulk symmetric electrolyte. For each $T^{*}$ below the critical temperature, the curves provide the two binodal values for the reduced total density.

The convexity properties of $f_{\text {tot }}$ are deduced from the phase diagram of Fig. 7: for a given reduced temperature $T^{*}$ below the critical temperature $T_{\text {crit }}^{*}$, the binodal points of $f_{\text {tot }}$, denoted by $\hat{\rho}_{\text {tot }}^{b}$ and $\hat{\rho}_{\text {tot }}^{\sharp}$, are determined from the liquid-vapor coexistence curves of Fig. 7. The approximate subset $\mathcal{K}^{* *}$ of the two-dimensional phase space then consists of two connected components (associated with the dilute and condensed phases) separated by 
the stripe $\left\{\hat{\rho}_{\text {tot }}^{b} \leq \hat{\rho}_{+}+\hat{\rho}_{-} \leq \hat{\rho}_{\text {tot }}^{\sharp}\right\}$ whose boundary are two parallel lines in the $\left(\rho_{+}, \rho_{-}\right)$plane. A comparison with the exact subset $\mathcal{K}^{* *}$ determined numerically by computing the double Legendre-Fenchel transform of $f$ (see [49]) shows relative differences in predicted ionic densities below $10^{-4}$ away from the interface and $10^{-2}$ near the interface.

5.2. Numerical method. Assuming (25) to be well-posed, we devise a numerical method to approximate its solution. Following the above discussion, we introduce the following regularization of the free energy functional:

$$
\mathcal{F}_{\eta}(\rho)=\mathcal{F}(\rho)+\frac{\eta^{2} L_{*}^{5}}{\beta} \sum_{i= \pm} \int_{\Omega} \frac{1}{2}\left|\nabla \rho_{i}\right|^{2},
$$

with nondimensional regularization parameter $\eta>0$. The constrained minimization problem (25) is then replaced by seeking ionic densities $\rho_{\eta}$ such that

$$
\left\{\begin{array}{l}
\rho_{\eta} \text { minimizes } \mathcal{F}_{\eta}\left(\rho_{\eta}\right) \text { defined by }(28), \\
\rho_{\eta} \text { satisfies the canonical constraint }(1) .
\end{array}\right.
$$

This problem is solved numerically using the techniques outlined in Sect. 3.1, where, in the Newton-Raphson iterations, the chemical potentials are evaluated using the convex hull $f^{* *}$ of the bulk free energy density.

We assume that, similarly to the simpler setting analyzed in [58], the singularly perturbed limit $\eta \rightarrow 0^{+}$represents a choice criterion between all minimizers of the nonconvex minimization problem (18) by sampling thermodynamically stable states where $f$ and $f^{* *}$ coincide. Solving (29) with a fixed positive value of the regularization parameter $\eta$ typically leads to a diffuse interface between the two phases, that is, the set $\mathcal{I}:=\left\{x \in \Omega ; \rho(x) \notin \mathcal{K}^{* *}\right\}$ has positive measure. The sharp interface approximation $\eta \rightarrow 0^{+}$leads to two distinct phases occupying the whole fluid domain $\Omega$, while the measure of $\mathcal{I}$ tends to zero. The parameter $\eta$ plays herein the role of a numerical parameter; from a physical viewpoint, a diffuse interface with a fixed value of $\eta$ can be more appropriate. We refer to [50] for further insight concerning the numerical methodology.

5.3. Results and discussion. Fig. 8 presents concentration profiles of counterions and coions as a function of the distance to the wall in the double-layer configuration $\left(L_{*}=1 \mathrm{~nm}\right)$. We consider a symmetric divalent (left panel) or trivalent (right panel) electrolyte with $\Sigma_{\mathrm{S}}=$ $0.2 \mathrm{C} \mathrm{m}^{-2}, 0.4 \mathrm{C} \mathrm{m}^{-2}$, and $0.6 \mathrm{C} \mathrm{m}^{-2}, \rho^{\text {salt }}=0.5 \mathrm{~mol} \mathrm{~L}^{-1}$, and $\sigma=2.27 \AA\left(T^{*}=0.0775\right)$. We observe that a single phase transition occurs within the pore, and that the position of the interface shifts toward the center as the surface charge density is increased. In all cases, the condensed phase contains most of the counterions and co-ions. In the divalent case with moderate ion diameter, the co-ion profile is non-monotone in the condensed phase, a feature that almost disappears in the trivalent case with large diameter. Fig. 9 presents the cloud of points $\left(\rho_{+}, \rho_{-}\right)$in the state space for the same parameters as in Fig. 8a. Each point corresponds to the value obtained at a node of the finite element mesh. For fixed $\Sigma_{\mathrm{S}}$, moving from right to left along a set of points corresponds to moving away from the charged wall. The two parallel oblique lines indicate the boundary of the subset $\mathcal{K}^{* *}$ in which the ionic densities are sought. We notice that the constraint that the ionic densities take values in the subset $\mathcal{K}^{* *}$ is very well satisfied by the present numerical method, up to very few mesh nodes (less than 0.1\%). 


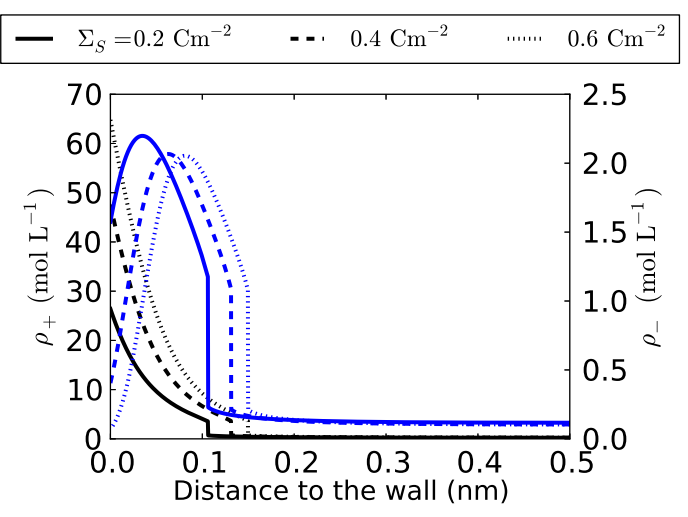

a

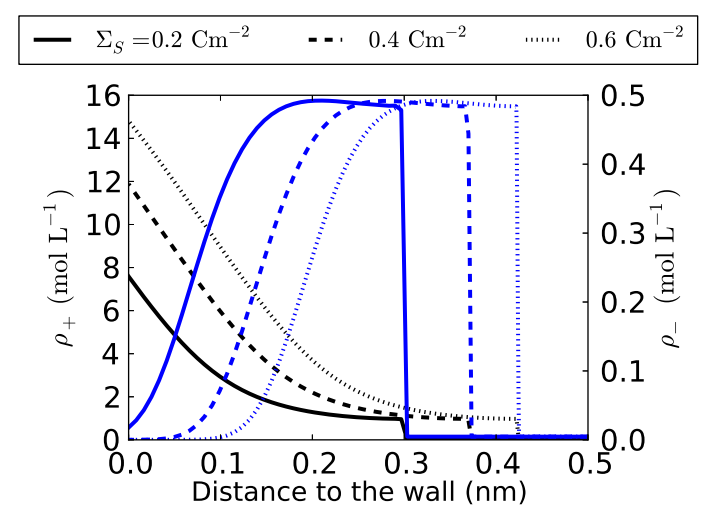

$\mathrm{b}$

Figure 8. (Color online) Concentration of counterions (black) and co-ions (blue) as a function of the distance to the wall for the double-layer configuration $\left(L_{*}=1 \mathrm{~nm}\right)$ with $\Sigma_{\mathrm{S}}=0.2 \mathrm{C} \mathrm{m}^{-2}, 0.4 \mathrm{C} \mathrm{m}^{-2}$, and $0.6 \mathrm{C} \mathrm{m}^{-2}$; (a) divalent electrolyte with $\rho^{\text {salt }}=0.5 \mathrm{~mol} \mathrm{~L}^{-1}$ and $\sigma=2.27 \AA\left(T^{*}=0.0775\right)$ and (b) trivalent electrolyte with $\rho^{\text {salt }}=0.2222 \mathrm{~mol} \mathrm{~L}^{-1}$ and $\sigma=4.5 \AA$ $\left(T^{*}=0.0705\right)$.

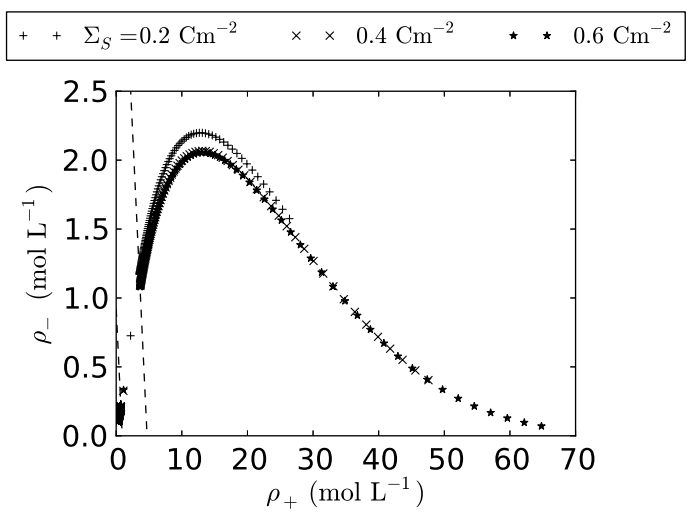

Figure 9. Double-layer configuration $\left(L_{*}=1 \mathrm{~nm}\right)$ for a symmetric divalent electrolyte with $\Sigma_{\mathrm{S}}=0.2$ and $0.6 \mathrm{C} \mathrm{m}^{-2}$, and $\rho^{\text {salt }}=0.5 \mathrm{~mol} \mathrm{~L}^{-1}$. Each point corresponds to ionic densities obtained at a certain node of the finite element mesh. The two parallel oblique lines indicate the boundary of the subset $\mathcal{K}^{* *}$.

Finally, to illustrate the capabilities of the numerical method to handle more complex geometries, Fig. 10 presents isocontours of counterion and co-ion concentrations for the configuration with circular inclusions with $L_{*}=1 \mathrm{~nm}, \Sigma_{\mathrm{S}}=0.2 \mathrm{C} \mathrm{m}^{-2}$, and $\rho^{\text {salt }}=0.5 \mathrm{~mol} \mathrm{~L}^{-1}$. We observe again the formation of a single interface whose shape closely follows that of the circular inclusion, and that the condensed phase contains most of the counterions and co-ions. 


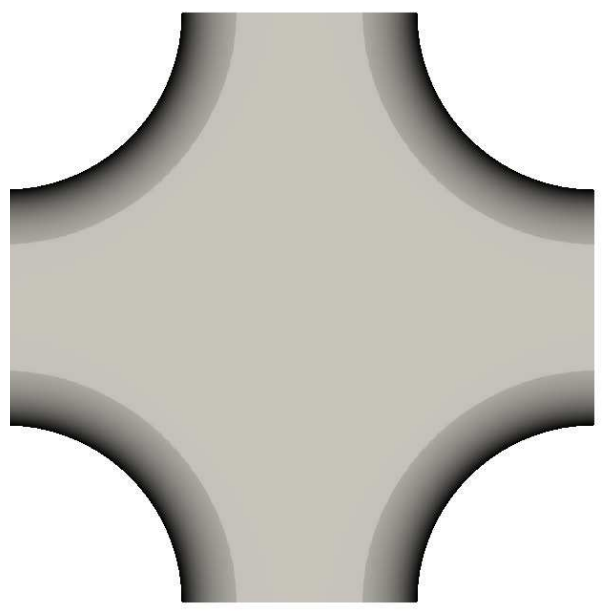

a

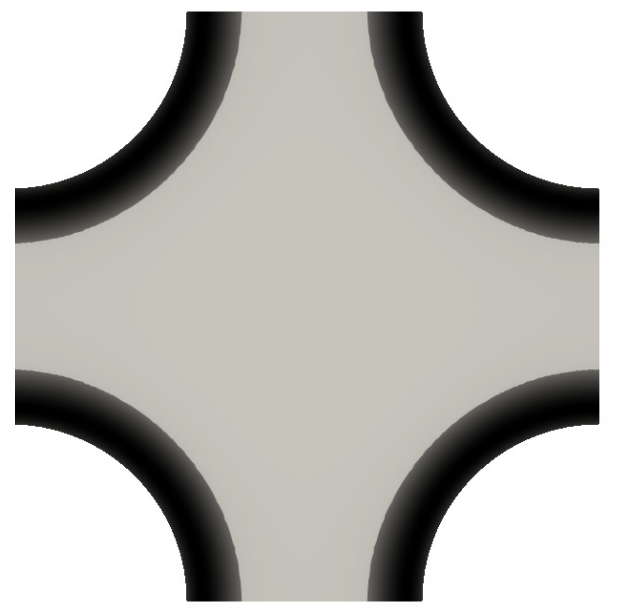

b

Figure 10. Circular inclusion within symmetric divalent electrolyte for $L_{*}=$ $1 \mathrm{~nm}, \Sigma_{\mathrm{S}}=0.2 \mathrm{C} \mathrm{m}^{-2}$, and $\rho^{\text {salt }}=0.5 \mathrm{~mol} \mathrm{~L}^{-1}$; concentration isocontours for (a) counterions (values between 0.27 and $25.3 \mathrm{~mol} \mathrm{~L}^{-1}$ ) and (b) co-ions (values between 0.09 and $1.82 \mathrm{~mol} \mathrm{~L}^{-1}$ ).

\section{Conclusions}

The main findings of the present study can be summarized as follows. Ionic concentration profiles are generally better described by PB than by DFT-MSA under the conditions investigated here, namely multi-valent ions under strong confinement, although DFT-MSA is able to capture the non-monotonicity of co-ion profiles missed by PB. The relatively accurate predictions obtained with PB in some situations can be partly attributed to the presence of added salt which tempers the effects of the confining double-layer. Furthermore, the failure of DFT-MSA for the lowest reduced temperatures considered herein is related to an incipient phase separation predicted by DFT-MSA at too high a critical temperature in the bulk. The conclusions regarding the pressure are quite different. Pressure predictions with DFT-MSA are more accurate than with $\mathrm{PB}$, since DFT-MSA reproduces pressure variations with the reduced temperature and, at the same time, yields values closer to those predicted by MC. These results confirm that nonideal effects are important to describe highly confined ionic solutions in thermodynamic equilibrium with a reservoir.

More elaborate models for nonideality can be incorporated into the present DFT in future work. Improved free energy functionals can be handled within the present numerical methodology if they are known in closed analytic form or in tabulated form on a sufficiently fine set of values for the ionic densities allowing for reasonably accurate data interpolation. These can include improvements in the description of electrostatic correlations near the critical point, of excluded volume effects near the solid surface (which can lead to attraction between likecharged surfaces), and of ion-solvent interactions especially for multi-valent ions and very small confinements. In isotropic media, accounting for Bjerrum's ion pairing improves the description of the coexistence curve and the value of the critical point. To better describe the strong electrostatic interactions between the ions and charged surfaces at low temperature, a formulation of the strong coupling theory has been obtained from the Wigner crystal [31]. An 
interpolation at short distance between the strong coupling and Poisson-Boltzmann theories should provide a better account of the correlations in the condensed ion profiles. Another interesting approach is to resort to a Weighted Density Approximation (WDA) instead of the LDA whereby a coarse-grained density obtained by convolution of the local density is used in the free energy; see, e.g., [40].

The present methodology, which is applicable to multi-dimensional geometries, can be of interest to study counterion condensation in confined ionic solutions in other applicative settings. As natural extensions of this work, one could now include the effect of nonideality in multi-scale approaches which currently use the PB theory as a starting point $[62,63,64$, 65]. Ionic fluids in the presence of random confinement can also be studied by sampling realizations of the geometric configuration of the nanopores and the results compared to previous work, e.g., [66]. Another important issue is that of dynamical properties, in particular the coupling of ion dynamics with fluid flows resulting in electro-kinetic effects [67]. The present DFT formulation can be used to include nonideality in numerical approaches which couple hydrodynamic flows to ionic fluxes via time-dependent DFT [68].

\section{ACKNOWLEDGMENTS}

Partial support by ANDRA (French National Agency for the Management of Nuclear Waste) is gratefully acknowledged; in particular, the first author has been supported through a PhD fellowship by ANDRA.

\section{REFERENCES}

[1] P. W. Debye and E. Hückel. The theory of electrolytes. I. lowering of freezing point and related phenomena. Phys. Z., 24:185, 1923.

[2] J. L. Lebowitz and E. Waisman. Mean spherical model integral equations for charged hard spheres. I. Method of solution. J. Chem. Phys., 56:3086-3093, 1972.

[3] J. L. Lebowitz and E. Waisman. Mean spherical model integral equations for charged hard spheres. II. Results. J. Chem. Phys., 56:3093-3099, 1972.

[4] L. Blum. Mean spherical model for asymmetric electrolytes. Mol. Phys., 30:1529-1535, 1975.

[5] L. Blum and J. S. Høye. Mean spherical model for asymmetric electrolytes. 2. Thermodynamic properties and the pair correlation function. J. Phys. Chem., 81:1311-1316, 1977.

[6] B. Groh, R. Evans, and S. Dietrich. Liquid-vapor interface of an ionic fluid. Phys. Rev. E., 57:6944-6954, 1998.

[7] N. K. Bjerrum. K. Dan. Vidensk. Selsk. Mat. Fys. Medd., 7:1, 1926.

[8] W. Ebeling and M. Grigo. Ann. Phys. (Leipzig), 37:21, 1980.

[9] W. Ebeling and M. Grigo. J. Sol. Chem., 11:151, 1982.

[10] M. Fisher and Y. Levin. Criticality in ionic fluids: Debye-Hückel theory, Bjerrum, and beyond. Phys. Rev. Lett., 71:3826, 1993.

[11] Y. Levin and M. Fisher. Criticality in the hard-sphere ionic fluid. Physica A, 225:164, 1996.

[12] J. Jiang, L. Blum, O. Bernard, J. M. Prausnitz, and S. I. Sandler. Criticality and phase behavior in the restricted-primitive model electrolyte: description of ion association. J. Chem. Phys., 116:7977, 2002.

[13] J. E. Flores-Mena, M. C. Barbosa, and Y. Levin. Criticality in confined ionic fluids. Phys. Rev. E, 63:066104, 2001.

[14] O. Pizio, A. Patrykiejew, and S. Sokolowski. Phase behavior of ionic fluids in slitlike pores: a density functional approach for the restricted primitive model. J. Chem. Phys., 121:11957, 2004.

[15] O. Pizio and S. Sokolowski. Phase behavior of the restricted primitive model of ionic fluids with association in slitlike pores. density-functional approach. J. Chem. Phys., 122:144707, 2005.

[16] M. Baus and J.-P. Hansen. Statistical mechanics of simple Coulomb systems. Phys. Rep., 59:1, 1980.

[17] M. Jardat, J.-F. Dufrêche, V. Marry, B. Rotenberg, and P. Turq. Salt exclusion in charged porous media: a coarse-graining strategy in the case of montmorillonite clays. Physical Chemistry Chemical Physics, 11(12):2023-2033, March 2009. 
[18] L. Dresner and K. A. Kraus. Ion exclusion and salt filtering with porous ion-exchange materials. J. Phys. Chem., 67:990, 1963.

[19] L. Dresner. The exclusion of ions from charged microporous solids. J. Phys. Chem., 69:2230, 1965.

[20] S. Engström and H. Wennerström. Ion condensation on planar surfaces. a solution of the PoissonBoltzmann equation for two parallel charged plates. J. Phys. Chem., 82:2711, 1978.

[21] G. H. Manning. Limiting laws and counterion condensation in polyelectrolyte solutions i. colligative properties. J. Chem. Phys., 51:924, 1969.

[22] M. Le Bret and B. H. Zimm. Distribution of counterions around a cylindrical polyelectrolyte and manning's condensation theory. Biopolymers, 23:287, 1984.

[23] E. Trizac, L. Bocquet, M. Aubouy, and H. H. von Grünberg. Alexander's prescription for colloidal charge renormalization. Langmuir, 19:4027-4033, 2003.

[24] P. Attard, D. J. Mitchell, and B. W. Ninham. Beyond Poisson-Boltzmann: images and correlations in the electrical double-layer. I Counterions only. J. Chem. Phys., 88:4987, 1988.

[25] R. Kjellander, S. Marcelja, and J. P. Quirk. Attractive double-layer interactions between calcium clay particles. Journal of Colloid and Interface Science, 126(1):194-211, November 1988.

[26] R. Kjellander, S. Marcelja, R. M. Pashley, and J. P. Quirk. Double-layer ion correlation forces restrict calcium-clay swelling. The Journal of Physical Chemistry, 92(23):6489-6492, November 1988.

[27] A. G. Moreira and R. R. Netz. Binding of similarly charged plates with counterions only. Phys. Rev. Lett., 87:078301, 2001.

[28] R. R. Netz. Electrostatistics of counter-ions at and between planar charged walls: From Poisson-Boltzmann to the strong-coupling theory. Eur. Phys. J. E, 5:557, 2001.

[29] A. Yu. Grosberg, T. T. Nguyen, and B. I. Shklovskii. The physics of charge inversion in chemical and biological systems. Rev. Mod. Phys., 74:329, 2002.

[30] L. Samaj and E. Trizac. Counterions at highly charged interfaces: From one plate to like-charge attraction. Phys. Rev. Lett., 106:078301, 2011.

[31] L. Samaj and E. Trizac. Wigner-crystal formulation of strong-coupling theory for counterions near planar charged interfaces. Phys. Rev. E, 84:041401, 2011.

[32] F. Paillusson and E. Trizac. Interaction regimes for oppositely charged plates with multivalent counterions. Physical Review E, 84(1):011407, July 2011.

[33] V. Kralj-Iglic and A. Iglic. A simple statistical mechanical approach to the free energy of the electric double layer including the excluded volume effect. J. Phys. II (France), 6:477-491, 1996.

[34] J.-P. Hansen, D. Goulding, and R. van Roij. Effective interactions between charged colloidal particles : Repulsion, attraction and phase separation. J. Phys. IV (France), 10:5, 1997.

[35] R. R. Netz and H. Orland. Field theory for charged fluids and colloids. Europhys. Lett., 45:726, 1999.

[36] J.-P. Hansen and H. Löwen. Effective interactions between electric double-layers. Annu. Rev. Phys. Chem., 51:209-242, 2000.

[37] D. Gillespie, W. Nonner, and R. S. Eisenberg. Density functional theory of charged, hard-sphere fluids. Phys. Rev. E., 68:2003, 031503.

[38] L. Mier-y Teran, S. H. Suh, H .S White, and H. T. Davis. A nonlocal free energy density functional approximation for the electrical double layer. J. Chem. Phys., 92:5087, 1990.

[39] H. Löwen, J.-P. Hansen, and P. A. Madden. Nonlinear counterion screening in colloidal suspensions. J. Chem. Phys., 98:3275-3289, 1993.

[40] A. Oleksy and J.-P. Hansen. Wetting and drying scenarios of ionic solutions. Mol. Phys., 109(7-10):1275$1288,2011$.

[41] H. Greberg, R. Kjellander, and T. Akesson. Ion-ion correlations in electric double layers from monte carlo simulations and integral equation calculations. Molecular Physics, 87(2):407-422, 1996.

[42] H. Greberg, R. Kjellander, and T. Akesson. Ion-ion correlations in electric double layers from monte carlo simulations and integral equation calculations part 2. case of added salt. Molecular Physics, 92(1):35-48, 1997.

[43] A. Delville and R. J. M. Pellenq. Electrostatic attraction and/or repulsion between charged colloids: A (NVT) monte-carlo study. Molecular Simulation, 24(1-3):1-24, 2000.

[44] Roland Kjellander, Alexander P. Lyubartsev, and Stjepan Marcelja. McMillan-Mayer theory for solvent effects in inhomogeneous systems: Calculation of interaction pressure in aqueous electrical double layers. The Journal of Chemical Physics, 114(21):9565-9577, June 2001. 
[45] R. J. M. Pellenq. On the origin of cement cohesion. Actualite Chimique, pages 12-22, March 2004. WOS:000220862400003.

[46] W. Kohn and L. J. Sham. Self-consistent equations including exchange and correlation effects. Phys. Rev., 140:A1133-A1138, 1965.

[47] A. Ern, R. Joubaud, and T. Lelièvre. Mathematical study of non-ideal electrostatic correlations in equilibrium electrolytes. Nonlinearity, 25:1635-1652, 2012.

[48] E. Luijten, M. E. Fisher, and A. Panagiotopoulos. University class of criticality in the Restricted Primitive Model electrolyte. Phys. Rev. Lett., 88:185701, 2002.

[49] L. Contento, A. Ern, and R. Vermiglio. An efficient algorithm for the double Legendre-Fenchel transform with application to phase separation. Technical report, University Paris-Est, CERMICS, 2013. hal .archives-ouvertes.fr/hal-00806597.

[50] R. Joubaud. Modélisation mathématique et numérique des fluides à l'échelle nanométrique. PhD thesis, University Paris-Est, 2012.

[51] W. van Megen and I. Snook. J. Chem. Phys., 73:4658-4662, 1980.

[52] G.M. Torrie and J.-P. Valleau. J. Chem. Phys., 73:5807-5816, 1980.

[53] B. Jönsson, H. Wennerström, and B. Halle. J. Phys. Chem., 1980.

[54] L. Guldbrand, B. Jönsson, H. Wennerström, and P. Linse. J. Chem. Phys., 80:2221-2228, 1984.

[55] J.-P. Valleau, R. Ivkov, and G.M. Torrie. J. Chem. Phys., 95:520-532, 1991.

[56] R.J.M. Pellenq, J.-M. Caillol, and Delville A. J. Phys. Chem. B, 101:8584-8594, 1997.

[57] L. Modica. The gradient theory of phase transitions and the minimal interface criterion. Arch. Ration. Mech. Anal, 98:123-142, 1987.

[58] Andrea Braides. $\Gamma$-convergence for Beginners. Oxford lecture series in mathematics, 2002.

[59] C.B. Muratov. Droplet phases in non-local Ginzburg-Landau models with Coulomb repulsion in two dimensions. Comm. Math. Phys., 299:45-87, 2010.

[60] R. Choksi and P. Sternberg. Periodic phase separation: the periodic isoperimetric and Cahn-Hilliard problems. Interfaces and Free Boundaries, 8:371-392, 2006.

[61] D. Goldman, C. Muratov, and S. Serfaty. The Gamma-limit of the two-dimensional Ohta-Kawasaki energy. I. Droplet density. Arch. Ration. Mech. Anal., 210 no. 2, 2013.

[62] C. Moyne and M. A. Murad. A two-scale model for coupled electro-chemo-mechanical phenomena and onsager's reciprocity relations in expansive clays: I homogenization analysis. Transport in Porous Media, 62(3):333-380, March 2006.

[63] C. Moyne and M. A. Murad. A two-scale model for coupled electro-chemo-mechanical phenomena and onsager's reciprocity relations in expansive clays: II computational validation. Transport in Porous Media, 63(1):13-56, April 2006.

[64] G. Allaire, A. Mikelić, and A. Piatnitski. Homogenization of the linearized ionic transport equations in rigid periodic porous media. J. Math. Phys., 51:123103, 2010.

[65] G. Allaire, R. Brizzi, J-F Dufrêche, A. Mikelić, and A. Piatnitski. Role of non-ideality for the ion transport in porous media: derivation of the macroscopic equations using upscaling. eprint arXiv:1309.3679, 2013.

[66] G. Pellicane, C. Caccamo, D. S. Wislon, and L. L. Lee. Replica Ornstein-Zernike self-consistent theory for mixtures in random pores. Phys. Rev. E, 69:061202, 2004.

[67] I. Pagonabarraga, B. Rotenberg, and D. Frenkel. Recent advances in the modelling and simulation of electrokinetic effects: bridging the gap between atomistic and macroscopic descriptions. Phys. Chem. Chem. Phys., 12(33):9566-9580, 2010.

[68] B. Rotenberg, I. Pagonabarraga, and D. Frenkel. Coarse-grained simulations of charge, current and flow in heterogeneous media. Faraday Discussions, 144(0):223-243, October 2009.

1: ANDRA, DRD/EAP, Parc De la Croix Blanche, 1,7 Rue Jean Monnet, 92298 ChâtenayMalabry CEDEX

2: University Paris-Est, Cermics (ENPC), 77455 Marne la Vallée cedex 2, France

3: Department of Mathematics, Imperial College London, SW7 2AZ London, UK 
4: Sorbonne Universités, UPMC Univ. Paris 06, UMr 8234 PHEniX, 75005 Paris, France

5: CNRS, UMR 8234 PHENIX, 75005 PARIS, FRANCE

6: Crmd, CnRS-Université D'Orléans, 1B rue de la Férollerie, 45071 Orléans Cedex 02, FRANCE 